\title{
Is it Crohn's disease? A severe systemic granulomatous reaction to sulfasalazine in patient with rheumatoid arthritis Leonard G Quallich ${ }^{1}$, Joel Greenson ${ }^{2}$, Hilary M Haftel ${ }^{1}$ and Robert J Fontana*1
}

Address: ${ }^{1}$ Department of Internal Medicine, University of Michigan Medical School, Ann Arbor, Michigan, 48109, USA and ${ }^{2}$ Department of Pathology, University of Michigan Medical School, Ann Arbor, Michigan, 48109, USA

E-mail: Leonard G Quallich - lquall@umich.edu; Joel Greenson - facjkgmd@umich.edu; Hilary M Haftel - hils@umich.edu; Robert J Fontana* - rfontana@umich.edu

*Corresponding author

This article is available from: http://www.biomedcentral.com/I47/-230X/I/8

(C) 200 I Quallich et al; licensee BioMed Central Ltd. Verbatim copying and redistribution of this article are permitted in any medium for any noncommercial purpose, provided this notice is preserved along with the article's original URL. For commercial use, contact info@biomedcentral.com

\begin{abstract}
Background: Sulfasalazine is a widely used anti-inflammatory agent in the treatment of inflammatory bowel disease and several rheumatological disorders. Although as many as $20 \%$ of treated patients may experience reversible, dose-dependent side effects, less frequent but potentially severe, systemic reactions have also been reported.

Case Presentation: A severe systemic reaction to sulfasalazine developed in a 21 -year old female with rheumatoid arthritis characterized by eosinophilia, granulomatous enteritis and myelotoxicity, cholestatic hepatitis, and seizures. The clinical course and management of this patient are presented as well as a review of the incidence and outcome of severe systemic reactions to sulfasalazine.

Conclusions: Granulomatous myelotoxicity and enteritis developed in a 21 year old female within 3 weeks of initiating sulfasalazine for rheumatoid arthritis. Following a short course of corticosteroids, the patient had resolution of her cholestatic hepatitis, rash, eosinophilia, and gastrointestinal symptoms with no residual manifestations at 7 months follow-up. Although severe reactions to sulfasalazine are rare and unpredictable, practicing physicians should be aware of unusual clinical presentations of toxicity when prescribing sulfasalazine.
\end{abstract}

\section{Background}

Sulfasalazine (SSZ) is a widely used and highly effective anti-inflammatory agent in the treatment of Crohn's disease and ulcerative colitis [1-3]. SSZ is also being used with increasing frequency in the treatment of various rheumatolgical disorders [4-7]. SSZ is preferred over other agents such as gold and penicillamine in rheumatology patients due to its more favorable side effect profile [8-10]. Nonetheless, rash, fever, and dyspepsia occur in $3 \%$ to $20 \%$ of treated patients $[7,11,12]$. These reactions are usually dose dependent and improve with dose reduction or discontinuation $[7,12]$. However, more serious but less common side effects have also been reported [13-19]. We now report a severe systemic reaction to SSZ in a 21-year-old female with rheumatoid arthritis characterized by granulomatous infiltration of the bone marrow, colon and small bowel as well as cholestatic hepatitis and seizures. 


\section{Case Report}

A 21-year old female was diagnosed with polyarticular rheumatoid arthritis in 1997 and was managed with naprosyn (Naproxen, Roche Laboratories, Nutley, NJ) for 4 years. Joint inflammation was well controlled with hydroxychlorquine (Plaquenil, Sanofi Pharmaceuticals, New York, NY) from October 1999 thru June 2000 when hydroxychlorquine was discontinued due to persistent anemia and a flare in her arthritis despite adequate therapy. The patient was started on iron supplementation and a 4-week course of low dose prednisone in June/ July 200o. Due to persistent arthritis, sulfasalazine was initiated on July 28, 2000 at a dose of $500 \mathrm{mg}$ twice dai$\mathrm{ly}$, increasing to $1000 \mathrm{mg}$ twice daily. Liver biochemistries were normal immediately before and two weeks after initiating SSZ. Three weeks after starting SSZ, the patient developed an erythematous rash over the upper extremities and trunk that improved with cetirizine (Zyrtec, Pfizer Inc, New York, NY). She then developed fatigue, abdominal pain, diarrhea, and night sweats which lead to hospitalization on 8/29/00 and SSZ was discontinued. At presentation, serum alkaline phosphatase was $923 \mathrm{IU} / \mathrm{L}$, AST $105 \mathrm{IU} / \mathrm{L}$, ALT $143 \mathrm{IU} / \mathrm{L}$, bilirubin $4.3 \mathrm{mg} / \mathrm{dl}$, and WBC was $9.6 \mathrm{k} / \mathrm{l}$ with $12 \%$ eosinophils. Over the next six days, she gained 20 pounds in fluid weight and had fevers of $103^{\circ} \mathrm{F}$ despite broad spectrum antibiotics. She was transferred on hospital day \# 6 to the University of Michigan Medical Center for further evaluation and management.

The patient reported a prior history of infectious mononucleosis at age 18 and was a non-smoker and consumed alcohol infrequently. She denied any known drug allergies or prior exposure to sulfa containing medications or antibiotics. There was no family history of allergy to sulfa products.

On hospital day \#6, the patient appeared toxic with a temperature of $102^{\circ} \mathrm{F}$, pulse $154 / \mathrm{min}$, and blood pressure of $100 / 58$. Scleral icterus was present as well as deeply jaundiced, edematous skin with a macular, erythematous rash. Non-tender, 1-2 cm lymph nodes were palpated in the neck, axilla, and groin. There were decreased breath sounds with mild bibasilar rales and a hyperdynamic precordium. The abdomen was diffusely tender and distended. Rectal examination revealed brown hemoccult + stool. Neurologic examination was unremarkable.

Laboratory studies revealed a WBC count of $44.2 \times 10^{6} /$ L with $11 \%$ bands, 24\% neutrophils, 30\% lymphocytes, $12 \%$ monocytes, and $22 \%$ eosinophils. The WBC and absolute neutrophil count nadired on hospital day \# 19 (Table 1). Hemoglobin was $9.3 \mathrm{~g} / \mathrm{dl}$, platelets 336,000 , reticulocyte count $3.1 \%$ and serum ferritin $17 \mathrm{ng} / \mathrm{ml}$. The serum alkaline phosphatase was $1002 \mathrm{IU} / \mathrm{L}$, AST $57 \mathrm{IU} /$ $\mathrm{L}$, ALT $76 \mathrm{IU} / \mathrm{L}$, total bilirubin $6.6 \mathrm{mg} / \mathrm{dl}$, albumin 1.6, total protein $3.6 \mathrm{~g} / \mathrm{dl}$ and INR 1.5. Serum fibrinogen was $126 \mathrm{mg} / \mathrm{dl}$ and d-dimer levels were elevated at 0.2-0.40 $\mathrm{mcg} / \mathrm{ml}$. Hepatitis A, B, and C serologies and ANA and ANCA were negative. CT scan of the chest, abdomen, and pelvis revealed bilateral pleural effusions, splenomegaly, and axillary, para-aortic, and inguinal lymphadenopathy. Stool Clostridium difficile toxin B was negative. Because of her persistent diarrhea, hypoalbuminemia, and anemia, an upper endoscopy and flexible sigmoidoscopy were performed on hospital day \# 8 to exclude a protein losing enteropathy. Although both studies were endoscopically normal, histopathology of random mucosal biopsies revealed characteristic features of Crohn's disease with granulomas in the colon and erosive duodenitis with a single granuloma in the small bowel (Figure 1). Anti-gliadin and anti-endomysial antibodies were negative. On hospital day \# 9, a bone marrow biopsy revealed marked eosinophilia and multiple, non-caseating granulomas. Flow cytometry of the bone marrow and peripheral blood revealed no evidence of malignancy. AFB and fungal stains of the gastrointestinal and bone marrow biopsies were negative as were cultures of the bone marrow 6 weeks later. A PPD and candida control were both nonreactive.

Given the suspicion of a systemic drug reaction as the etiology of her illness, the patient was given high dose intravenous steroids for three days starting on hospital day \# 14 followed by a prednisone taper. Within 24 hours, the patient had a rapid improvement of her diarrheal symptoms followed by resolution of her peripheral edema and lymphadenopathy. There were also concomitant improvements in her hematological and liver profiles (Table 1, Figure 2). However, the patient developed generalized absence seizures on hospital day \#20. Cerebrospinal fluid analysis revealed an isolated increased WBC of 14 with a normal protein level. An MRI of the brain with gadolinium contrast identified patchy and irregular signal enhancement in the gray matter of the occipital poles, frontoparietal regions, and cerebellar hemispheres. The patient was started on phenytoin and later changed to oxcarbazepine. At 1 month post-discharge, the patient was markedly improved as her steroid dose was tapered. At 3 months post-discharge, a repeat upper and lower endoscopy with terminal ileum intubation and random biopsies were normal. At 7 months after presentation off steroids, the patient had completely recovered with no evidence of residual hematological, gastrointestinal, or neurological sequelae.

\section{Discussion}

Sulfasalazine is a widely used anti-inflammatory agent in the treatment of Crohn's disease and ulcerative colitis 


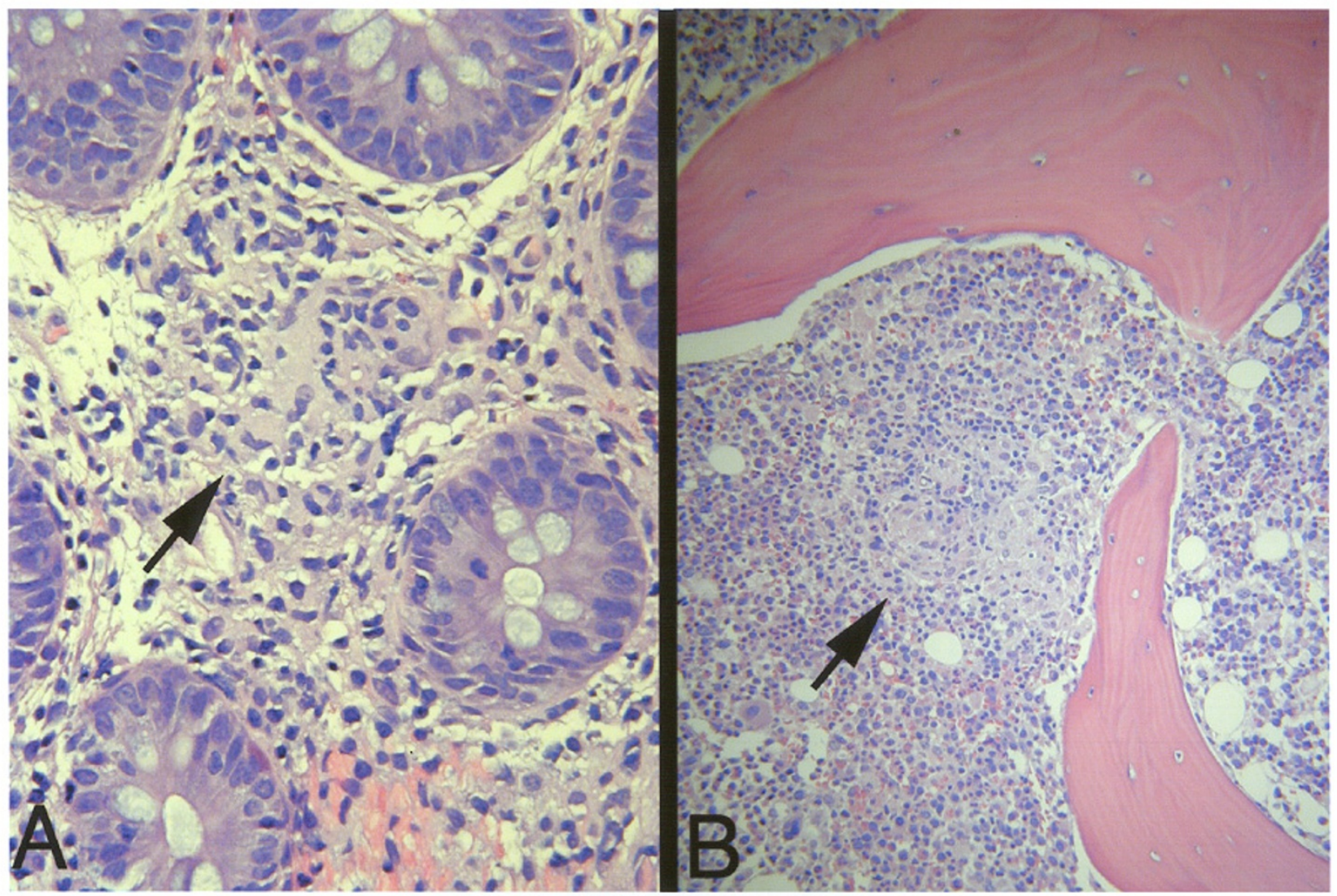

\section{Figure I}

A: Medium-power photomicrograph of the colon showing a small epithelioid granuloma (arrow) (Hematoxylin and eosin stain. B: Low-power photomicrograph of bone marrow showing a hypercellular marrow with an epithelioid granuloma (arrow) (Hematoxylin and eosin stain).

Table I: Hematologic parameters

\begin{tabular}{cccc}
\hline Hospital Day & $\begin{array}{c}\text { Total WBC } \\
\text { Count }\end{array}$ & $\begin{array}{c}\text { Absolute } \\
\text { Neutrophil } \\
\text { Count }\end{array}$ & $\begin{array}{c}\text { Absolute } \\
\text { Eosinophil } \\
\text { Count }\end{array}$ \\
\hline-14 & 6,000 & 5,000 & 200 \\
$\mathbf{1}$ & 9,600 & 5,000 & $1,152^{* *}$ \\
$\mathbf{6}$ & 45,200 & 15,500 & $9,700^{* *}$ \\
$\mathbf{1 4}$ & 10,100 & 3,800 & $5,400^{* *}$ \\
$\mathbf{1 8}$ & 5,300 & 1700 & 300 \\
$\mathbf{1 9}$ & 6,000 & $1000^{*}$ & $500^{* *}$ \\
$\mathbf{2 0}$ & 5,000 & 2400 & 200 \\
$\mathbf{4 0}$ & 11,600 & 9600 & 200 \\
\hline
\end{tabular}

\footnotetext{
*Absolute neutropenia $($ ANC < $1500 / \mathrm{ml}):{ }^{* *}$ Absolute eosinophilia $\left(\right.$ AEC $>400 / \mathrm{ml}$ ) ${ }^{\dagger}$ Day $-14=$ Baseline values 2 weeks after starting SSZ Day $\mathrm{I}=$ Admission to Hospital Day $40=$ Follow-up after discharge
}

[1-3]. Recent data has demonstrated that SSZ is also safe and effective in the treatment of rheumatoid arthritis and other rheumatological disorders [4,5]. Sulfasalazine is a dimer composed of 5 -aminosalicylic acid linked by an azo bond to sulfapyridine. The anti-inflammatory activity of SSZ is due to the 5-aminosalicylic acid component while the majority of the adverse side effects have been attributed to the sulfapyridine moiety [14-16]. Fever, abdominal pain, and rash may develop in 3\% to $20 \%$ of treated patients, but these side effects are usually mild and improve with dose reduction $[7,11,12]$. Severe side effects necessitating SSZ discontinuation occur in less than $5 \%$ of treated patients [11].

Our patient developed a severe systemic illness following three weeks of SSZ therapy for rheumatoid arthritis. In retrospect, her clinical presentation with rash, fever, and lymphadenopathy within 4 weeks of initiating treatment is consistent with severe SSZ toxicity [17]. However, in this case and others, it can be difficult to distinguish a se- 


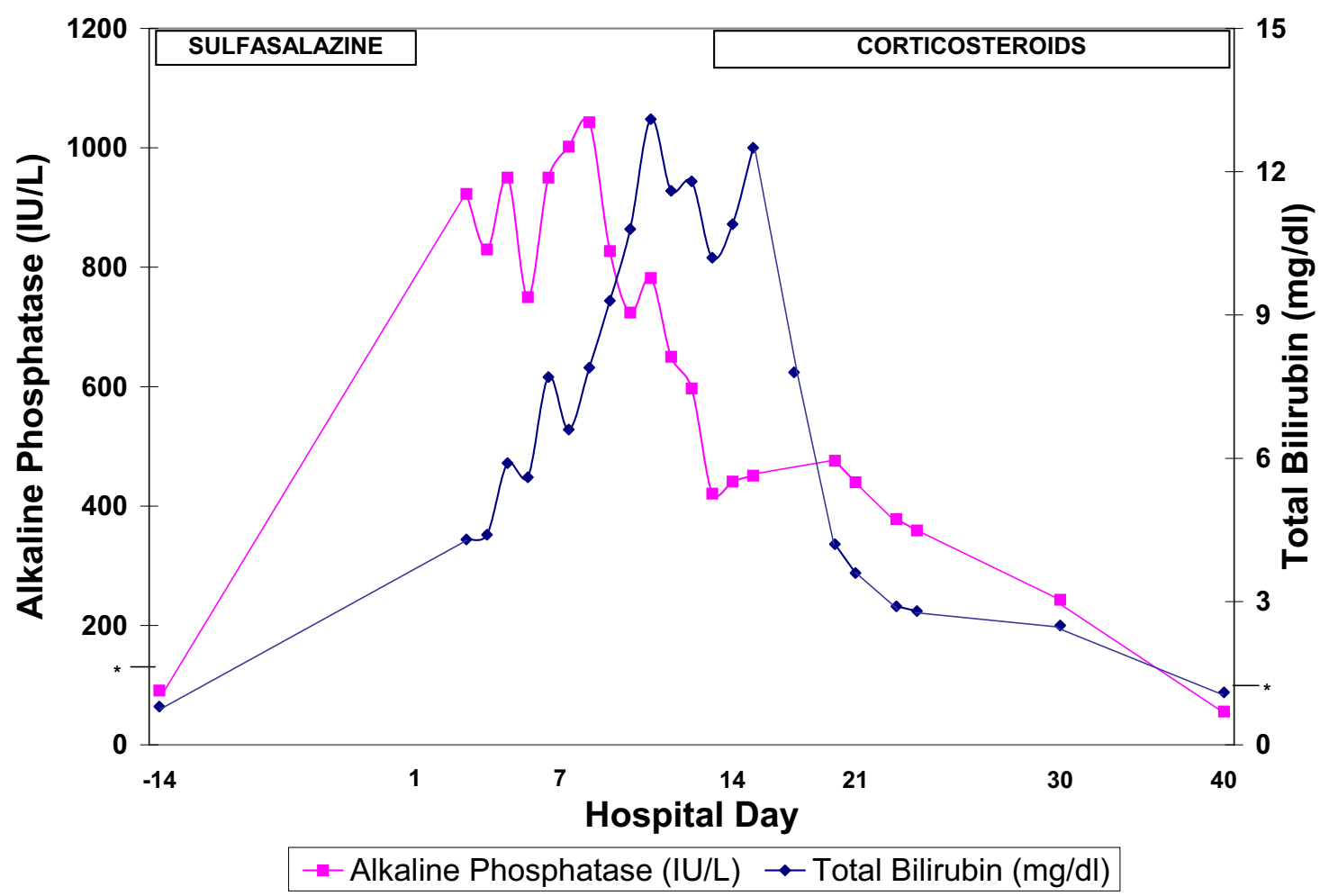

Figure 2

Liver biochemical profile. The patient had a normal serum alkaline phosphatase and bilirubin level 14 days prior to presentation. The marked intrahepatic cholestasis did not improve until initiation of steroids on day \#l4. At post-discharge follow-up on day \#40, both the serum alkaline phosphatase and bilirubin had normalized. ${ }^{*}=$ Upper limit of normal

vere drug reaction from other disease processes such as infection and malignancy. In our patient's case, a concern of lymphoma was raised in light of her underlying rheumatoid arthritis as well as prominent lymphadenopathy and splenomegaly. In addition, a possible opportunistic fungal, mycobacterial, or other atypical infection was raised with the high fevers, low grade DIC, and disseminated granulomas that persisted after SSZ discontinuation. Lastly, Crohn's disease or ulcerative colitis with extraintestinal manifestations was a possibility with her severe iron deficiency anemia, diarrhea, and granulomatous enteritis. It was only after extensive evaluation that a preliminary diagnosis of SSZ drug toxicity was made. Occult inflammatory bowel disease was not definitively excluded until follow-up endoscopy was repeated 3 months later.
This patient's clinical presentation with multi-organ involvement due to an immunoallergic reaction to SSZ has not been previously reported. The gastrointestinal mucosal biopsies in this patient demonstrated multiple noncaseating granulomas with prominent eosinophilia suggestive of Crohn's disease (Figure 1). In addition, the patient's bone marrow biopsy also demonstrated multiple non-caseating granulomas and eosinophilia. Although other systemic illnesses such as untreated tuberculosis, $Q$ fever, fungal infections, and lymphoma can lead to gastrointestinal granuloma, the acute nature of this patient's symptoms and rapid improvement with steroids make these causes unlikely. Furthermore, special stains and studies of the patient's tissues revealed no evidence of malignancy or infection. To our knowledge, only one other case of a systemic granulomatous reaction to SSZ 
has been reported which improved within 24 hours of SSZ discontinuation [18]. In other cases of granulomatous drug reactions, multi-organ involvement with fevers and eosinophilia have been reported $[19,20]$.

Our patient had severe cholestatic hepatitis with coagulopathy. Although Still's disease can rarely lead to hepatic involvement with cholestatic hepatitis, the prior history of rheumatoid factor seropositivity and a low serum ferritin level made this diagnosis unlikely [21]. Granulomatous hepatitis with marked elevations in serum alkaline phosphatase and granulomas on liver biopsy have been reported in patients with SSZ hepatotoxicity [22-24]. Although liver tissue was not obtained in our patient, the predominance of serum alkaline phosphatase and bilirubin level elevations and the presence of granulomas in other tissues suggest that she probably had granulomatous hepatitis. The presumed mechanism of liver injury in SSZ hepatotoxicity is an idiosyncratic hypersensitivity response. Both severe hepatitis and acute liver failure have been attributed to SSZ [25-28]. Patients with SSZ hepatotoxicity demonstrate a prompt recurrence of symptoms upon rechallenge with SSZ or sulfonamide [15,29-31]. Susceptibility to the toxic effects of SSZ and other sulfonamides may be due to the interaction of metabolic pathways regulating $\mathrm{N}-$ acetylation and specific detoxification of the metabolites of the drug $[32,33]$. However, it remains unclear if genetic differences in drug metabolizing enzyme expression may increase the risk of SSZ toxicity.

Myelotoxicity in this patient was manifest by leukopenia and neutropenia (Table 1). SSZ has previously been associated with neutropenia with an estimated incidence of $0.04 \%$ during the first 30 days of treatment [34]. Bone marrow biopsy specimens of patients with SSZ induced agranulocytosis reveal a paucity of myeloid cells but full recovery typically occurs within 10 days of drug discontinuation [35-37]. Although our patient had adequate myeloid cells in her bone marrow biopsy, she developed absolute neutropenia by hospital day \#19 that improved with steroid therapy. SSZ induced agranulocytosis is believed to be mediated by immunological hypersensitivity of myeloid precursors [38]. Spontaneous recovery from SSZ agranulocytosis is frequently seen, although fatalities have been reported and some patients may benefit from granulocyte macrophage-colony stimulating factor $[39,40]$.

A final clinical feature of SSZ toxicity in our patient was the development of seizures. Although this patient had no known risk factors, the onset of seizures began in combination with other clinical manifestations of SSZ toxicity. Interestingly, the MRI demonstrated diffuse gray matter abnormalities. Although central nervous system vasculitis due to immune complex deposition or drug-induced lupus is a possible etiology of seizures in this patient, there was no evidence of immune complex deposition elsewhere. Another possibility is that this patient may have developed CNS granulomas. Seizures have previously been reported in patients receiving SSZ, often in the setting of other clinical manifestations of toxicity $[31,41,42]$. Although a brain biopsy would be needed to prove our hypotheses, there is a precedent for other systemic granulomatous disorders such as sarcoidosis leading to CNS granuloma formation and seizures $[43,44]$. Fortunately, this patient has remained stable with no evidence of a progressive neurological disorder 7 months after presentation.

Treatment of severe adverse drug reactions is primarily supportive once a diagnosis has been established and the offending agent has been discontinued. However, in this patient's case with her prominent features of a hypersensitivity reaction and failure to improve after SSZ discontinuation, corticosteroids were administered to hasten recovery. Fortunately, most of her clinical symptoms promptly improved after steroids were begun. The use of steroids in the setting of SSZ toxicity is controversial and not always effective. In cases of fulminant hepatic failure and agranulocytosis, several patients have died despite the use of steroids $[27,28,37]$. Intravenous immunoglobulin was reported as a successful "rescue" therapy in a patient with SSZ induced fulminant hepatic failure and erythroid hypoplasia who had not responded to steroids [25]. Due to the infrequent occurrence of severe SSZ toxicity that does not improve with drug discontinuation, it is unlikely that randomized controlled trials of steroids or other interventions will be performed. It appears reasonable to reserve steroids for patients with severe systemic SSZ toxicity that fail to respond to supportive care following drug discontinuation.

\section{Conclusions}

Sulfasalazine is a commonly used and effective medication in the treatment of inflammatory bowel disease and various rheumatologic disorders. Side effects usually develop within 3 months of treatment and are frequently managed with dose adjustments. However, there is a subset of patients who may develop severe and unusual systemic side effects. Although rheumatology patients may be at increased risk of SSZ toxicity compared to IBD patients, there are no reliable laboratory or clinical predictors of SSZ toxicity [45]. Therefore, practicing physicians should be aware of the potential for severe, unexplained granulomatous reactions to SSZ that can mimic other infectious, malignant, and autoimmune conditions. Fever, rash, and eosinophilia developing within 3 months of initiating SSZ should alert the clinician to the possibility of a drug reaction. Patients with 
SSZ toxicity should avoid exposure to all sulfonamide containing drugs due to potential cross-reactivity. In addition, some inflammatory bowel disease patients have demonstrated cross-reactivity to 5-ASA products [11]. If severe symptoms do not resolve with SSZ discontinuation, a short course of corticosteroids may be of benefit.

\section{Competing interests}

None declared

\section{References}

I. Peppercorn MA: Sulphasalazine pharmacology, clinical use, toxicity, and related new drug development. Ann Intern Med 1984, 3:377-386

2. Das KM: Sulphasalazine therapy in inflammatory bowel disease. Gastroenterol Clin North Amer 1989, I 8:1-20

3. Dick AP, Grayson MJ, Carpenter RG, Petrie A: Controlled trial of sulfasalazine in the treatment of ulcerative colitis. Gut 1964, 5:437-432

4. Dougados M, Van der Linden S, Leirisalo-Repo M, et al: Sulfasalazine in the treatment of sponyloarthropathy: a randomized, multi-center, double-blinded, placebo-controlled study. Arthritis Rheum 1995, 38:618-627

5. Pinals RS, Kaplan SB, Lawson JG, Hepburn B: Sulfasalazine in rheumatoid arthritis. Arthritis Rheum 1986, 29:I427-I434

6. Imundo LF, Jacobs JC: Sulfasalazine therapy for juvenile rheumatoid arthritis. J Rheumatol 1996, 23:360-366

7. Amos RS: The history of the use of sulphasalazine in rheumatology. Br J Rheumatol 1995, 34:2-6

8. Van Riel PLCM, Van Gestel AM, Van De Putte LBA: Long-term usage and side effect profile of sulphasalazine in rheumatoid arthritis. $\mathrm{Br}$ / Rheumatol I 995, 34:40-42

9. Amos RS, Pullar TT, Bax DE, et al: Sulphasalazine for rheumatoid arthritis: toxicity in $\mathbf{7 7 4}$ patients monitored for one to I I years. Br Med J (Clin Res Ed) 1986, 293:420-423

10. Situnayake RD, Grindulis KA, McConkey B: Long-term treatment of rheumatoid arthritis with sulphasalazine, gold, or penicillamine: a comparison using life-table methods. Ann Rheum Dis 1987, 46:177-183

I I. Nakajima H, Munakata A, Yoshida : Adverse effects of sulfasalazine and the treatment of ulcerative colitis with mesalazine. J Gastroenterol 1995, 30:1 I5- I I7

12. American College of Rheumatology Ad Hoc Committee on Clinical Guidelines: Guidelines for monitoring drug therapy in rheumatoid arthritis. Arthritis Rheum 1996, 39:723-731

13. Laasila K, Leirisalo-Repo M: Side effects of sulphasalazine in patients with rheumatic diseases of inflammatory bowel disease. Scand I Rheumatol 1994, 23:338-340

14. Watkinson G: Sulphasalazine: A review of $\mathbf{4 0}$ years' experience. Drugs 1986, 32:1-11

I5. Losek JD, Werlin SL: Sulfasalazine hepatotoxicity. Am J Dis Child 1981, 135:107|-1072

16. Dujovne $C$, Chan $C$, Zimmerman J: Sulfonamide hepatic injury. $N$ Engl J Med 1967, 277:785-788

17. Brooks H, Taylor H, Nichol F: The three week sulphasalazine syndrome. Clin Rheum 1992, I I:566-568

18. Rafoth R: Systemic granulomatous reaction to salicylazosulfapyridine (Azulfidine) in a patient with Crohn's disease. Dig Diseases 1974, 19:465-469

19. Singer DR, Simpson JG, Catto GR, Johnston AW: Drug hypersensitivity causing granulomatous interstitial hepatitis. Am J Kid Dis 1988, I I:357-359

20. Bramlet DA, Posalaky Z, Olson R: Granulomatous hepatitis as a manifestation of quinidine hypersensitivity. Arch Intern Med 1980, I 40:395-397

21. Takami A, Nakao S, Miyamori H, et al: Adult Onset Still's disease with submassive hepatic necrosis. Internal Medicine 1995, 34:8991

22. Fich A, Schwartz J, Braverman D, Zifroni A, Rachmilewitz D: Sulfasalazine hepatotoxicity. Am J Gastroenterol I 984, 79:40 I-402
23. Namias A, Bhalotra $R$, Donowitz M: Reversible sulfasalazine-induced granulomatous hepatitis. J Clin Gastroenterol I981, 3:193198

24. Callen J, Soderstrom : Granulomatous hepatitis associated with salicylazosulfapyridine therapy. S Med Journal 1978, 71:II591160

25. Huang JL, Hung I, Chen LC, Lee WY, Hsueh C, Hsieh KH: Successfully treated sulphasalazine-induced fulminant hepatic failure, thrombocytopenia and erythroid hypoplasia with intravenous immunoglobulin. Clin Rheumatol 1998, I 7::349-352

26. Rubin R: Sulfasalazine-induced fulminant hepatic failure and necrotizing pancreatitis. Am / Gastroenterol 1994, 89:789-79|

27. Marinos G, Riley J, Painter D, McCaughan G: Sulfasalazine-induced fulminant hepatic failure. / Clin Gastroenterol I992, I4: I32-I 35

28. Ribe J, Benkov K, Thung S, Shen S, LeLeiko N: Fatal massive hepatic necrosis: a probable hypersensitivity reaction to sulfasalazine. Am J Gastroenterol 1986, 8 I:205-208

29. Kanner RS, Tedesco FJ, Kalser MH: Azulfidine (sulfasalazine) induced hepatic injury. Am J Dig Dis 1978, 23:956-8

30. Gulley RM, Mirza A, Kelley CE: Hepatotoxicity of salicylazosulfapyridine: $\mathbf{A}$ case report and review of the literature. $\mathrm{Am} J$ Gastroenterol 1979, 72:561-564

31. Smith MD, Gibson GE, Rowland R: Combined hepatotoxicity and neurotoxicity following sulfasalazine administration. Aust N Z J Med 1 982, I 2:76-80

32. Shear NH, Spielberg SP, Grany DM, et al: Differences in metabolism of sulfonamides predisposing to idiosyncratic toxicity. Ann Intern Med 1986, 105:I79-184

33. Rieder MJ, Shear NH, Kanee A, et al: Prominence of slow acetylator phenotype among patients with sulfonamide hypersensitivity reactions. Clin Pharm Ther 1991, 49:13-17

34. Keisu M, Ekman E: Sulfasalazine associated agranulocytosis in Sweden 1972-1989. Eur J Clin Pharmacol 1992, 43:215-218

35. Maddocks JL, Slater DN: Toxic epidermal necrolysis, agranulocytosis and erythroid hypoplasia associated with sulfasalazine. J R Soc Med 1980, 73:587-588

36. Roth DA, Lindert M: A fatal case of Azulfidine-induced agranulocytosis. Gastroenterology 1959, 37:787-789

37. Jacobson IM, Kelsey PB, Blyden GT, et al: Sulfasalazine induced agranulocytosis. Am J Gastroenterol 1985, 80:I I8-I2I

38. Jick $H$, Myers MW, Dean AD: The risk of sulfasalazine and mesalazine-assoicated blood disorders. Pharmacotherapy 1995 , I5:|76-18|

39. Kuipers E, Vellenga E, Joost T, Hazenberg B: Sulfasalazine induced agranulocytosis treated with granulocyte-macrophage colony stimulating factor. J Rheumatol 1992, 19:62। -622

40. Roddie P, Dorrance $H$, Cook M, Rainey J: Treatment of sulphasalazine-induced agranulocytosis with granulocyte macrophage-colony stimulating factor. Aliment Pharm Ther 1995, 9:711-712

4I. Senturk T, Aydintug A, Duzgun N, Tokgoz G: Seizures and hepatotoxicity following sulphasalazine administration. Rheumatol Int 1997, 17:75-77

42. Hill ME, Gordon C, Situnayake RD, Heath DA: Sulfasalazine induced seizures and dysphagia. J Rheumatol 1994, 2 I:748-749

43. Krumholz A, Stern BJ, Stern EG: Clinical implications of seizures in neurosarcoidosis. Arch Neurol I991, 48:842-844

44. Pentland B, Mitchell JD, Cull RE, Ford MJ: Central nervous system sarcoidosis. QJM 1985, 56:457-465

45. Wolkenstein P, Charue D, Laurent $P$, et al: Metabolic predisposition to cutaneous adverse drug reactions: Role in toxic epidermal necrolysis caused by sulfonamides and anticonvulsants. Arch Derm 1995, I 3 1:544-55 I 